\title{
A Local-Search Based Heuristic for the Unrestricted Block Relocation Problem
}

\author{
Dominique Feillet, Sophie N. Parragh† Fabien Tricoire ${ }^{\ddagger}$
}

\begin{abstract}
The unrestricted block relocation problem is an important optimization problem encountered at terminals, where containers are stored in stacks. It consists in determining the minimum number of container moves so as to empty the considered bay following a certain retrieval sequence. A container move can be either the retrieval of a container or the relocation of a certain container on top of a stack to another stack. The latter types of moves are necessary so as to provide access to containers which are currently not on top of a stack. They might also be useful to prepare future removals. In this paper, we propose the first local search type improvement heuristic for the block relocation problem. It relies on a clever definition of the state space which is explored by means of a dynamic programming algorithm so as to identify the locally optimal sequence of moves of a given container. Our results on large benchmark instances reveal unexpectedly high improvement potentials (up to 50\%) compared to results obtained by state-of-the-art constructive heuristics.
\end{abstract}

\section{Introduction}

Container terminals are hubs connecting waterborne, rail, and road transportation networks. A more pronounced modal shift towards more sustainable means of transport, such as from road to rail or water, will put even more emphasis on their efficient operations. Within container terminals, containers awaiting further movement are usually stored in stacks. This is due to restricted amounts of space, as well as to restricted operating areas of handling equipment, such as gantry cranes. Very often, the sequence in which containers arrive is completely different from the sequence in which they need to be retrieved; moreover, it is not possible to take into account the sequence in which they will be retrieved while they arrive. This entails that in order to retrieve a container which does not end up on top of a stack, other containers have to be moved (to other stacks) in the same bay. Assuming the sequence in which the stacked containers need to be retrieved to be known, the aim is to minimize the number of unproductive movements (i.e., the number of reshuffles). The underlying optimization

\footnotetext{
*Ecole des Mines de Saint-Etienne and LIMOS UMR CNRS 6158, CMP Georges Charpak, Gardanne, F-13541 France, feillet@emse.fr

${ }^{\dagger}$ Institute of Production and Logistics Management, Johannes Kepler University Linz, Austria, sophie.parragh@jku.at

${ }^{\ddagger}$ Institute of Production and Logistics Management, Johannes Kepler University Linz, Austria, fabien.tricoire@jku.at
} 
problem is known as the container relocation problem (CRP) (Forster and Bortfeldt, 2012) or the block(s) relocation problem (BRP) (Caserta et al., 2011) in the literature. In its basic version, first introduced by Kim and Hong (2006), unproductive movements may only concern those containers which are on top of the container which needs to be retrieved next. It is often referred to as the restricted container (or block) relocation problem (R-BRP) (see, e.g., Galle et al., 2018). In this paper we study the BRP without such a restriction. This means that, in anticipation of the retrieval sequence, unproductive relocations may concern containers on top of any of the stacks. In the literature, this version is usually referred to as the unrestricted BRP (U-BRP) and several authors have proposed heuristic as well as exact solution methods to solve the U-BRP. In this paper, we propose the first local search based improvement method for the U-BRP.

The BRP has received considerable attention in the literature. For a literature review and a classification scheme considering all kinds of problems arising in situations where containers are organized in stacks, we refer to Lehnfeld and Knust (2014). This review covers the literature until 2014. Therefore, we will focus here on those works which have appeared later than that. We will first give a brief overview of contributions which study the R-BRP and we will then discuss recent works on the unrestricted version in further detail.

Among the more recent works on the restricted BRP is the one of Jovanovic and Voß (2014). They propose a chain heuristic which introduces a look-ahead feature and is thus able to improve on the results of basic greedy algorithms developed previously. More recently, Expósito-Izquierdo et al. (2015) have proposed a mathematical model for the R-BRP and a branch-and-bound algorithm which can be truncated and thus used as a heuristic. Eskandari and Azari (2015) propose corrections and improvements to one of the formulations proposed in Caserta et al. (2012) and present improved results for the R-BRP and $\mathrm{Ku}$ and Arthanari (2016b) develop an exact method which relies on clever ways to reduce the search space as well as on a bi-directional search scheme. Very recently, Galle et al. (2018) have propose a new mathematical formulation relying on a binary encoding of different configurations.

For the unrestricted BRP studied in this paper, Jin et al. (2015) propose a look-ahead heuristic, in which a tree search algorithm is called so as to anticipate the impact of the next relocation. They are able to improve on the results of Forster and Bortfeldt $(2012)$ and Zhu et al. (2012).

Tricoire et al. (2018) design a branch-and-bound algorithm and several heuristics for the $\mathrm{U}$-BRP, relying on the notion of safe moves and decreasing sequences, and incorporate them into metaheuristic search schemes which they call rake search and pilot method. The branchand-bound algorithm relies on a new lower bound, improving on the one of Forster and Bortfeldt (2012), and it employs the loop idea of Tanaka and Mizuno (2015), in which the lower bound is iteratively increased by one.

Very recently, Tanaka and Mizuno (2018) have designed a branch-and-bound algorithm for the unrestricted BRP. It relies on new dominance properties concerning partial sequences of relocations which do not appear in optimal solutions, as well as an improved lower bound. Benchmark instances with at most 10 stacks and 50 items are consistently solved to optimality with stacking height restrictions within 30 minutes of computation time.

Also Azari et al. (2017) rely on a branch-and-bound based heuristic algorithm. In contrast to other studies, they aim at minimizing the total working time of the crane. 
New lower bounds for the U-BRP have been proposed and used in an A* type algorithm by Quispe et al. (2018). The first new bound is a combinatorial lower bound, the second relies on pattern databases.

The pre-marshalling problem concerns the re-ordering of containers stored in stacks such that no reshuffling is necessary during retrieval. New unifying problem formulations of the premarshalling problem, the R-BRP and the U-BRP have recently been developed by de Melo da Silva et al. (2018). While the new formulations are more efficient than previous mathematical programming based approaches the authors acknowledge that tailored branch-and-bound algorithms still provide superior performance in terms of run time as well as number of instances solved to optimality.

The pre-marshalling problem is also subject to investigation in Jovanovic et al. (2017). The authors propose a multi-heuristic method, which includes a correction step. This correction step detects consecutive relocations of the same container and merges them. The idea underlying this step is similar to what motivated us to develop a local search algorithm.

Prandtstetter (2013) developed a dynamic programming based branch-and-bound algorithm for the pre-marshalling problem. To reduce the number of states, a heuristic rule for determining equivalent states is introduced and used to obtain a heuristic algorithm.

Very recently Tanaka and Tierney (2018) have proposed a new branch-and-bound algorithm for the pre-marshalling problem solving a number of previously unsolved instances to optimality. Parreño Torres and Alvarez-Valdes (2019) develop new integer programming models as well as an iterative solution method which, in each iteration, increases the lower bound on the maximum number of moves needed to rearrange the bays by one until a feasible and thus optimal solution has been found.

Generalizations of the BRP involve, e.g., the work of Hakan Akyüz and Lee (2014), who introduce the dynamic container relocation problem in which containers are received and retrieved from a single yard-bay. They propose three different heuristics. $\mathrm{Ku}$ and Arthanari (2016a) propose to incorporate departure time windows and they develop a stochastic dynamic programming model, since the retrieval sequence of containers belonging to the same departure time window is unknown.

Summarizing the above, it becomes clear that, currently, three streams of research exist. The first stream focuses on the development of efficient mathematical models, the second stream concerns the design of exact methods, mostly based on the branch-and-bound concept, while the third stream of research focuses on the identification of heuristic rules to obtain good but not necessarily optimal solutions quickly. In this paper we open up a fourth stream of research: we propose a local search based heuristic algorithm for the U-BRP, relying on dynamic programming, which allows to improve any heuristically generated solution. It locally reoptimizes the sequence of unproductive moves (or relocations) of a given container, with the objective of reducing their number. Relocations are not modified for other containers.

The remainder of the paper is organized as follows. Section 2 gives a more detailed problem definition. In Section 3 we present our local search based improvement heuristic. Numerical experiments are detailed and analyzed in Section 4 . Section 5 concludes the paper and draws some possible perspectives. 


\section{Problem definition}

We now provide a definition of the U-BRP. Given a set of $N$ blocks or containers which are stored in $W$ stacks of maximum height $H_{\max }$ in a bay, the aim is to retrieve the containers according to a certain sequence. This sequence is represented by consecutive numbers from 1 to $N$ assigned to the containers, where container number 1 has to be retrieved first and container number $N$ last. A container can only be retrieved if it sits on top of a stack; if there are other containers sitting above it, they must be moved to other stacks first. Moving a container to another stack is what we call a relocation. The aim is to minimize the total number of relocations $R$ necessary to retrieve all containers. The total number of container movements is then given by $N+R$ (retrieval plus relocation movements). We call configuration the layout of the bay at a given stage of the removal process. Each move (relocation or removal of a container) induces a new configuration. These configurations are numbered from 1 to $N+R+1$, where configuration 1 represents the initial layout of the bay and configuration $N+R+1$ the final (empty) layout. Note that this final configuration is numbered $N+R+1$ because it results from $N$ retrievals and $R$ relocations. A solution $\mathcal{S}$ to the U-BRP represents the sequence of $N+R+1$ configurations.

\section{A local search algorithm for the U-BRP}

As explained in Section 1, our new local search operator $O P T(n)$ reoptimizes the relocations of container $n$ without modifying the moves of any other container $(1 \leq n \leq N)$. The locally best sequence of moves for container $n$ is computed by dynamic programming. Given a starting solution $\mathcal{S}$, we repeatedly apply $O P T(n)$ for all containers, until a local optimum is reached. The local search algorithm (LS) is detailed in Algorithm 1.

In order to avoid useless calls to $\operatorname{OPT}(n)$, a lower bound $L B_{n}$ is precomputed for each container. It gives the minimal number of moves required for the container. This number is 1 if this container blocks another container with higher priority, 0 otherwise. A container is skipped when its number of relocations in $\mathcal{S}$ (denoted $f_{n}$ ) is equal to $L B_{n}$ (Line 5).

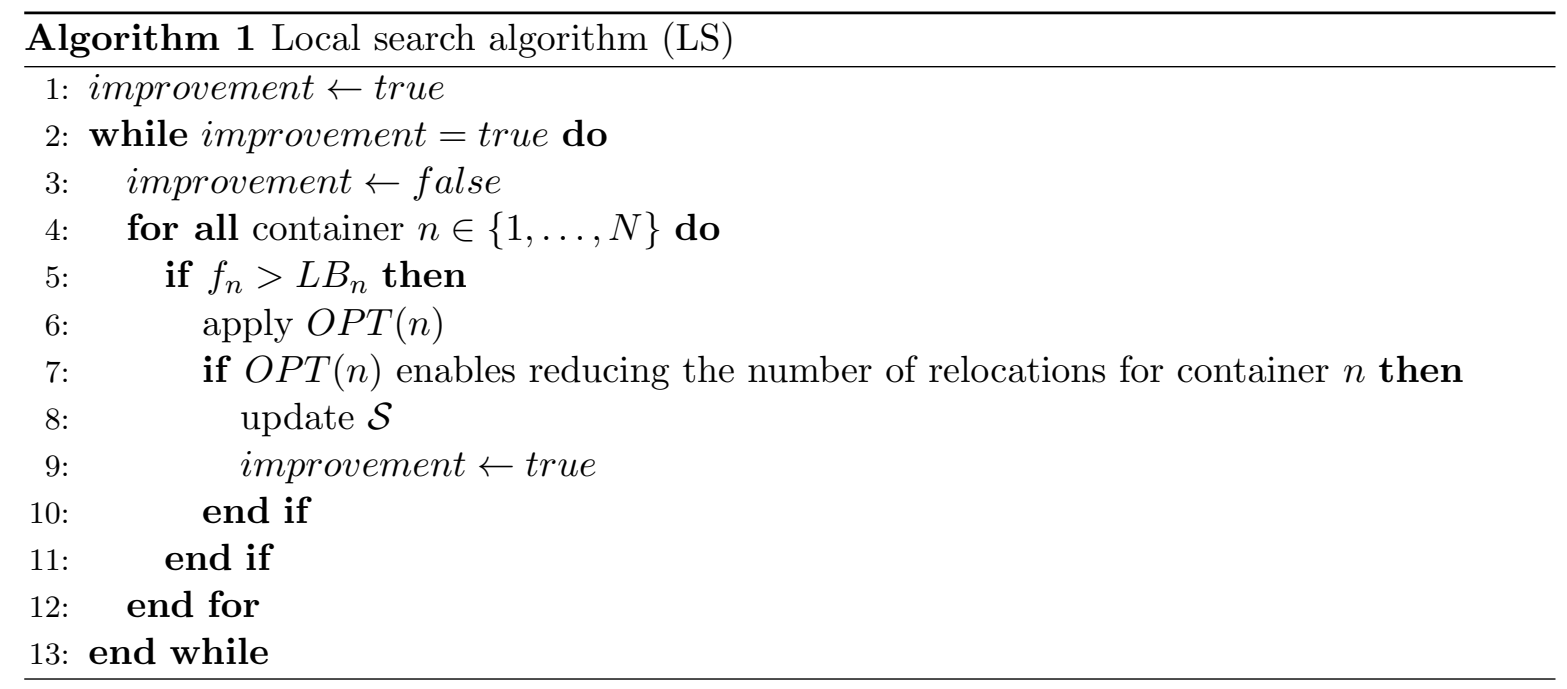


In what follows, we first detail the state space defined by our new local search operator $\operatorname{OPT}(n)$ (Section 3.1). Thereafter, in Section 3.2, we precise how the best path in this statespace is computed.

\section{$3.1 \quad$ State-space}

In this section, we introduce the state space on which dynamic programming is applied. Let $\mathcal{S}$ be a BRP solution on which we apply $\operatorname{OPT}(n)$. Recall that $\mathcal{S}$ represents the $N+R+1$ bay configurations, each defined by the retrieval or relocation of one container, starting from the initial bay configuration and ending with an empty bay.

We call step the transition from a configuration to the next in solution $\mathcal{S}$. Steps are numbered from 1 to $N+R$. Step $k$ allows to pass from configuration $k$ to configuration $k+1$. We denote the operation constituting a transition or step $k$ by $\sigma^{k}=\left(\sigma_{1}^{k}, \sigma_{2}^{k}\right)$, where $\sigma_{1}^{k}$ denotes the original stack of the container which is moved (in configuration $k$ ) and $\sigma_{2}^{k}$ its new stack (in configuration $k+1$ ), $\sigma_{2}^{k}=$ "-" in case of a retrieval.

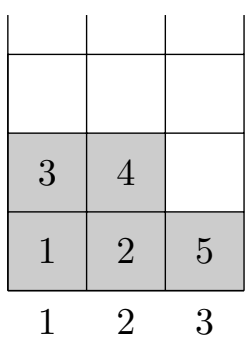

Configuration 1

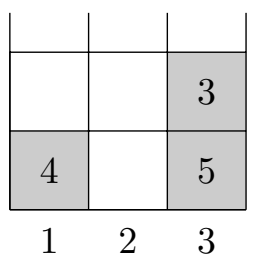

Configuration 6
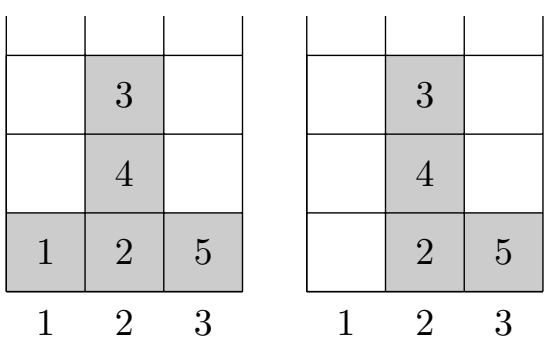

$\begin{array}{lll}1 & 2 & 3\end{array}$

Configuration 2

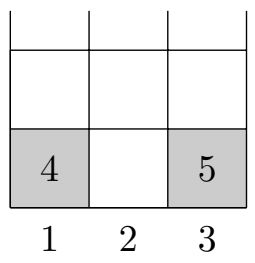

Configuration 7

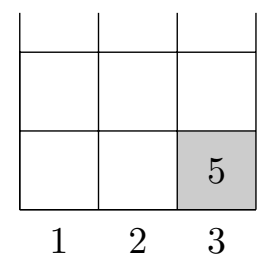

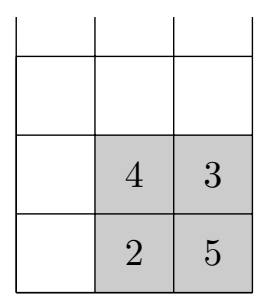
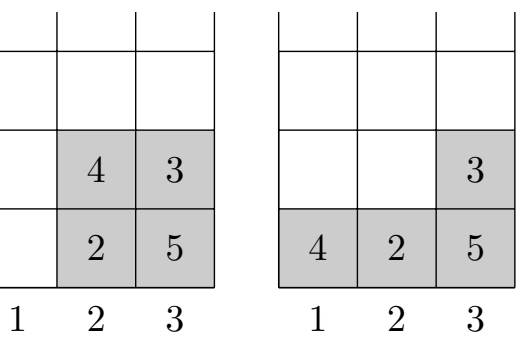

$1 \quad 2 \quad 3$

Configuration 4 Configuration 5

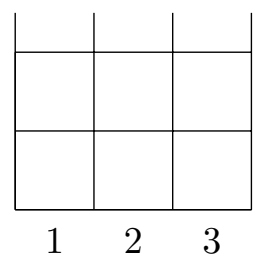

Configuration 8 Configuration 9

Figure 1: Configurations of a given solution $\mathcal{S}$ for a BRP instance with $W=3$ and $N=5$.

\begin{tabular}{ccc}
\hline & Step & $\left(\sigma_{1}, \sigma_{2}\right)$ \\
\hline 1 & Relocate 3 & $(1,2)$ \\
2 & Retrieve 1 & $(1,-)$ \\
3 & Relocate 3 & $(2,3)$ \\
4 & Relocate 4 & $(2,1)$ \\
\hline
\end{tabular}

\begin{tabular}{ccc}
\hline & Step & $\left(\sigma_{1}, \sigma_{2}\right)$ \\
\hline 5 & Retrieve 2 & $(2,-)$ \\
6 & Retrieve 3 & $(3,-)$ \\
7 & Retrieve 4 & $(1,-)$ \\
8 & Retrieve 5 & $(3,-)$ \\
\hline
\end{tabular}

Table 1: Steps for the solution $\mathcal{S}$ of Figure 1

Figure 1 and Table1 1 illustrate the concepts of configuration and steps for a given solution of a small BRP instance. 


\subsubsection{Removal of container $n$ from solution $\mathcal{S}$}

We introduce a sequence of configurations and steps, that we call $\mathcal{S}^{-n}$, and whose definition is detailed subsequently. Intuitively, $\mathcal{S}^{-n}$ only considers the part of solution $\mathcal{S}$ that occurs before the retrieval of container $n$ and modifies this sub-solution by removing container $n$ from the bay. More precisely, $\mathcal{S}^{-n}$ is obtained by the following constructive algorithm:

1. $\mathcal{S}^{-n}$ is initialized with no configuration;

2. Configurations and steps of solution $\mathcal{S}$ are appended to $\mathcal{S}^{-n}$ :

- starting from configuration 1 and going up to the last configuration before the retrieval of container $n$,

- ignoring configurations that result from the relocation of container $n$.

3. Container $n$ is then removed from all these configurations, i.e., for each configuration:

- the position where $n$ is located is first emptied,

- the tier of every container located above $n$ is decreased by 1 .

Steps are defined accordingly. Figure 2 depicts the configurations and steps of $\mathcal{S}^{-3}$ for the example of Figure 1. Configurations 1 to 6 of Figure 1 are considered, in this order. Configurations 2 and 4 are skipped because they follow the relocation of container 3 . In the four remaining configurations, container 3 is removed.
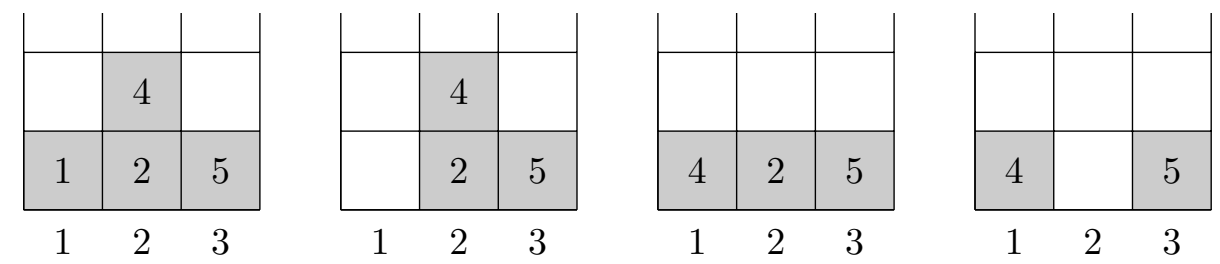

\begin{tabular}{cc}
\hline Step & $\left(\sigma_{1}, \sigma_{2}\right)$ \\
\hline 1 & $(1,-)$ \\
2 & $(2,1)$ \\
3 & $(2,-)$ \\
\hline
\end{tabular}

Figure 2: $\mathcal{S}^{-3}$ for solution $\mathcal{S}$ of Figure 1

We denote by $M$ the number of configurations in $\mathcal{S}^{-n}$. These configurations are numbered from 1 to $M$, and the steps between these configurations from 1 to $M-1(M=4$ in the example of Figure 2].

We denote by $h(s, t)$ the height of stack $s$ in configuration $t$ of $\mathcal{S}^{-n}$. For example, in the configurations depicted in Figure 2, $h(2,1)=h(2,2)=2, h(2,3)=1$ and $h(2,4)=0$.

\subsubsection{Definition of the state space graph}

The state space graph is defined by states (nodes) and transitions (arcs) between these states. 
States (nodes) of the state-space are named $\operatorname{STATE}(t, s, h)$, where $t \in\{1, \ldots, M\}$ indicates a configuration in $\mathcal{S}^{-n}, s \in\{1, \ldots, W\}$ indicates a stack in the bay and $h \in\left\{1, \ldots, H_{\max }\right\}$ a tier in this stack.

$S T A T E(t, s, h)$ represents a bay configuration based on configuration $t$ of solution $\mathcal{S}^{-n}$, modified by inserting container $n$ in stack $s$ and at tier $h$. The container initially located at position $(s, h)$ in configuration $t$ and containers above have their tier augmented by 1 . Figure 3 gives some examples of states.

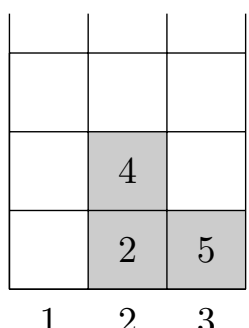

Configuration 2

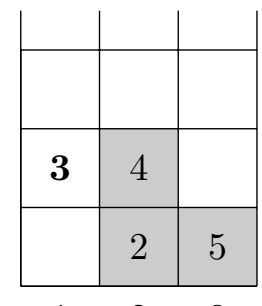

$\begin{array}{lll}1 & 2 & 3\end{array}$

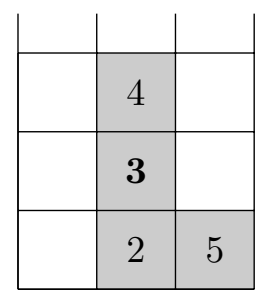

$1 \quad 2 \quad 3$

$\operatorname{STATE}(2,2,2)$

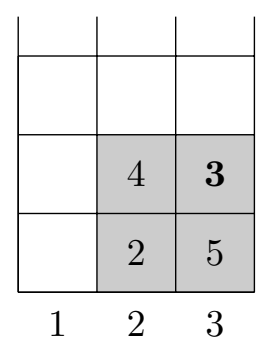

$\operatorname{STATE}(2,3,2)$

Figure 3: Some examples of states for configurations of $\mathcal{S}^{-3}$ depicted in Figure 2

A state $\operatorname{STATE}(t, s, h)$ is said to be feasible if the following conditions hold.

- States with $2 \leq t \leq M-1$ :

1. Container $n$ is not floating above other containers: $h \leq h(s, t)+1$;

2. A slot is available in the stack to insert container $n: h(s, t)<H_{\max }$;

3. Container $n$ is not on top of the container that is to be moved in the next step: if $\sigma_{1}^{t}=s, h \leq h(s, t)$

4. Container $n$ does not complete the stack that receives a container at the next step: if $\sigma_{2}^{t}=s, h(s, t)+1<H_{\max }$.

- States with $t=1$ :

The only feasible state is $\operatorname{STATE}\left(1, s_{n}^{0}, h_{n}^{0}\right)$ that represents the initial layout of the bay, where $\left(s_{n}^{0}, h_{n}^{0}\right)$ are the initial coordinates of $n$. This state is called initial state.

- States with $t=M$ :

1. Container $n$ is exactly on top of a stack: $h=h(s, t)+1$;

2. A slot is available in this stack: $h(s, t)<H_{\max }$.

Feasible states with $t=M$ are thus those for which container $n$ can be retrieved. These states are called final states.

For example, the last two states on Figure 3 are feasible, but the first one is not. 
Transitions (arcs) between states are defined as follows. Given a feasible $S T A T E(t, s, h)$, transitions from this state are only defined to a subset of feasible states of the next configuration, that is, states of the form $S T A T E\left(t+1, s^{\prime}, h^{\prime}\right)$. They consist of relocating (or not) container $n$ and applying step $t$ of solution $\mathcal{S}^{-n}$.

Let us consider a feasible state $\operatorname{STATE}(t, s, h)$ with $t<M$. Transitions from this state are defined as follows:

- A transition of cost 0 to $S T A T E(t+1, s, h)$ is added if $S T A T E(t+1, s, h)$ is feasible.

- If $h=h(s, t)+1$, a transition of cost 1 to $\operatorname{STATE}\left(t+1, s^{\prime}, h\left(s^{\prime}, t+1\right)+1\right)$ is added for all stacks $s^{\prime} \in\{1, \ldots, S\} \backslash\{s\}$ if $\operatorname{STATE}\left(t+1, s^{\prime}, h\left(s^{\prime}, t+1\right)+1\right)$ is feasible.

The first case corresponds to a strategy where container $n$ is not relocated and step $t$ is simply applied. In the second case, container $n$ is relocated to stack $s^{\prime}$ before applying step $t$.

The optimal set of relocations for container $n$ is then obtained by computing a shortest path in the graph defined by this state space, from the initial state $\operatorname{STATE}\left(1, s_{0}^{0}, h_{n}^{0}\right)$ to any of the final states.

The state-space graph for our example is given in Figure 4. Obviously, from $\operatorname{STATE}(3,1,1)$ no transition to a feasible final state $(t=M, M=4)$ is possible.

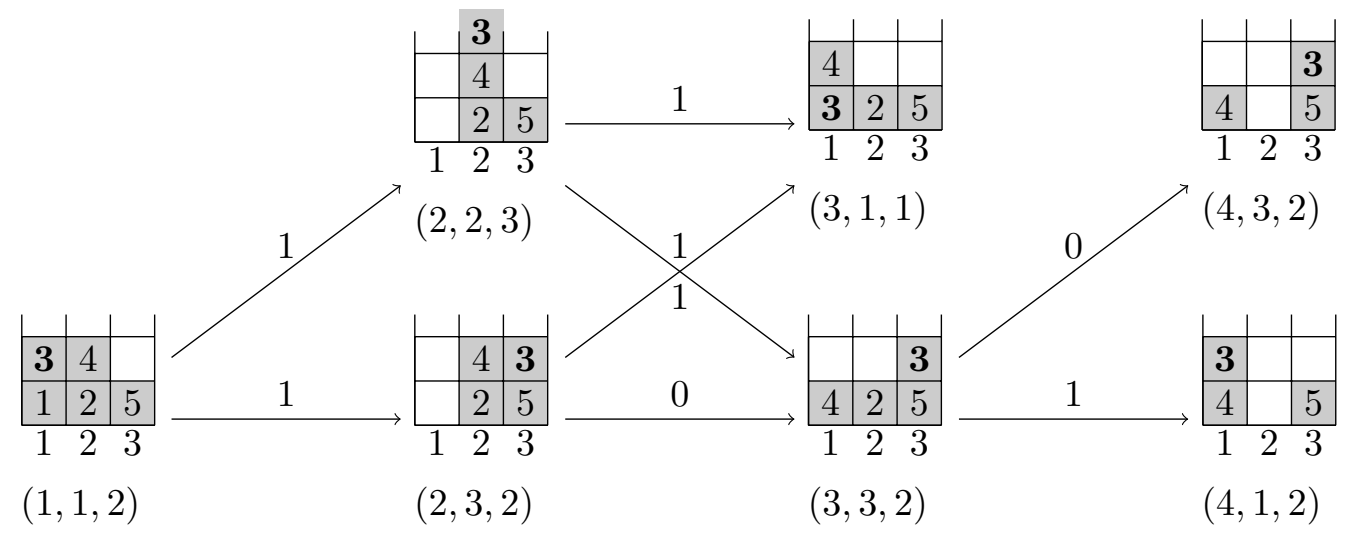

Figure 4: The state space graph for optimizing the relocations of container 3 of solution $\mathcal{S}$ given in Figure 1

\subsection{Local search operator $O P T(n)$ : exploration}

In the following, we explain the algorithm to explore the defined state-space so as to identify the best sequence of relocations for a given item. Thereafter, we discuss the implemented speedups. 


\subsubsection{Algorithm}

To find the min-cost path in the state-space graph, this graph is not explicitly constructed. Instead, we perform a breadth-first search from the initial state. The algorithm is detailed in Algorithm 2 and explained hereafter.

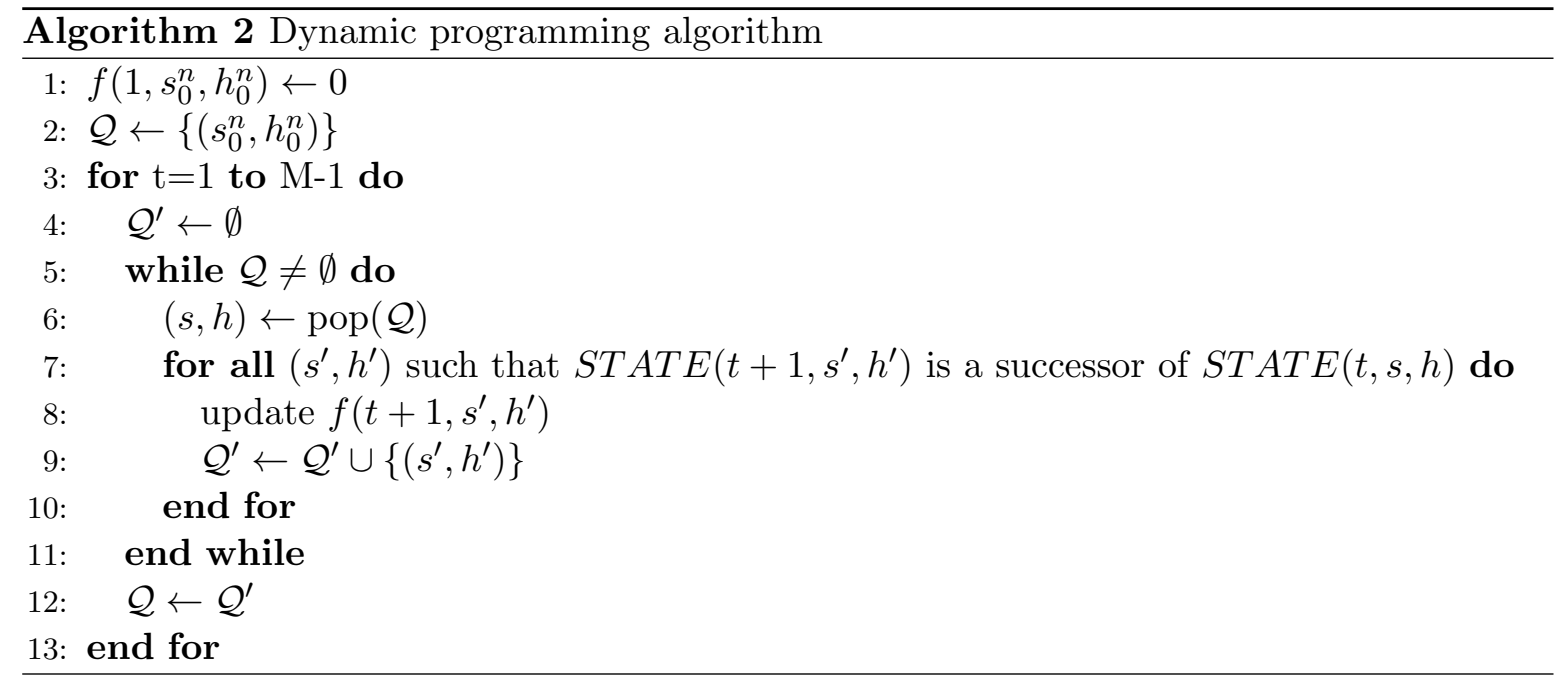

Function $f$ represents the current minimal cost to reach a state. It is initialized to 0 for the initial state. A loop considers all the states that have been reached for a given value of $t$ and determines the reachable states for $t+1$. In order to do so, two queues $\mathcal{Q}$ and $\mathcal{Q}^{\prime}$ are used. $\mathcal{Q}$ contains the feasible states for configuration $t$. $\mathcal{Q}^{\prime}$ is initially empty and progressively enriched with feasible successors of states in $\mathcal{Q}$. When $\mathcal{Q}^{\prime}$ is complete, i.e., all feasible successor states have been generated, $\mathcal{Q}^{\prime}$ is copied to $\mathcal{Q}$ and the procedure is repeated for the next configuration. At line 8, the value associated with a state is updated if a better path reaching this state has been found.

In order to evaluate in constant time if a state is feasible, values $h(s, t)$ are precomputed for all stacks $s$ and configurations $t$. At Line 7, all stacks $s^{\prime}$ are tried for the generation/improvement of states; given $s^{\prime}$, a single value $h^{\prime}$ obtained from $h\left(s^{\prime}, t\right)$ is considered. The complexity of the loop (Lines 7 10 ) is thus $W$ times the complexity of the update function. In order to achieve a complexity $O(1)$ for this function, two matrices of size $W \times H_{\max }$ are introduced. The matrices store state information for configurations $t$ and $t+1$, respectively. With these matrices, $f\left(t+1, s^{\prime}, h^{\prime}\right)$ can be obtained in $O(1)$. The two matrices are reinitialized at the end of every iteration, when $t$ is increased.

Queue $\mathcal{Q}$ has a maximal size equal to $W \times H_{\max }$. All the elements in $\mathcal{Q}$ can a priori be transferred to $\mathcal{Q}^{\prime}$ without an additional relocation, but only those elements with container $n$ on top of a stack allow a relocation. The worst-case complexity of an iteration (Lines 5 to 11) is thus $O\left(W \times H_{\max }+W^{2}\right)$, i.e., $O\left(W \times \max \left(H_{\max }, W\right)\right)$. The worst-case complexity of Algorithm 2 is finally $O\left(M \times W \times \max \left(H_{\max }, W\right)\right)$. 


\subsubsection{Speedups}

In order to reach a maximal efficiency for our local search operator, we implemented several speedup techniques:

Upper bound. As the operator aims at decreasing the number of relocations for container $n$, states that can only be reached with a large number of relocations of container $n$ are not interesting. Let $f_{n}$ be the number of relocations of $n$ in solution $\mathcal{S}$. If $f\left(t+1, s^{\prime}, h^{\prime}\right) \geq f_{n}$, $\left(s^{\prime}, h^{\prime}\right)$ is not added to $\mathcal{Q}^{\prime}$ at Line 9. Additionally, if $f\left(t+1, s^{\prime}, h^{\prime}\right) \geq f_{n}-1$ and state $\left(M, s^{\prime}, h^{\prime}\right)$ is not feasible, $\left(s^{\prime}, h^{\prime}\right)$ is also not added to $\mathcal{Q}^{\prime}$. Indeed, under these conditions, one more relocation is needed for container $n$ before it can be retrieved.

Useless evaluations. Applying a step $t$ of $\mathcal{S}^{-n}$ only modifies stacks $\sigma_{1}^{t}$ and $\sigma_{2}^{t}$. Thus, relocations of container $n$ for a given value of $t$ can be limited to the following two cases (except for $t=1$ ):

1. Container $n$ is located in stack $\sigma_{1}^{t-1}$. Then all relocations should be tried: they could not be performed at the previous steps.

2. Container $n$ is located in another stack. Then, relocations for $n$ should be tried towards two stacks: $\sigma_{1}^{t-1}$ and $\sigma_{2}^{t-1}$. Other stacks have not been modified and acceptable relocations have already been generated.

Aspiration. Every time that the value of a state is updated (Line 8), an attempt to generate a final state is done. The principle is to identify if the state could be prolongated to a feasible final state without any relocation. Three conditions are evaluated:

- $f\left(t+1, s^{\prime}, h^{\prime}\right) \leq f_{n}-1$,

- $h\left(s^{\prime}, t^{\prime}\right)<H_{\max }$ for $t^{\prime} \geq t+1$,

- $h\left(s^{\prime}, t^{\prime}\right) \geq h^{\prime}-1$ for $t^{\prime} \geq t+1$.

The first condition ensures that the state would lead to an improvement. The second condition checks if there is room for container $n$ in stack $s^{\prime}$ until it is retrieved. The third condition makes sure that containers below $n$ in stack $s^{\prime}$ will not be moved until the retrieval (the height of the stack is always larger). If the three conditions hold, the dynamic programming algorithm is stopped. The sequence of relocations that leads to $\left(t+1, s^{\prime}, h^{\prime}\right)$ is returned.

Note that the first two speedups do not modify the result of the algorithm. The aspiration speedup on the contrary can modify the sequence of relocations returned by the local search procedure. Furthermore, this sequence is not necessarily optimal: condition $f\left(t+1, s^{\prime}, h^{\prime}\right) \leq$ $f_{n}-1$ ensures that the sequence is improving but some shorter paths might exist in the state-space.

Introducing the upper bound has no impact on the worst-case complexity of the algorithm. With the Useless evaluations mechanism, the worst-case complexity of Lines 5 11 falls to $O\left(W \times H_{\max }+W\right)$ and that of Algorithm 2 to $O\left(M \times W \times H_{\max }\right)$. The conditions for aspiration can be computed in constant time with a simple preprocessing phase computing values $\min _{t^{\prime} \geq t} h(s, t)$ and $\max _{t^{\prime} \geq t^{\prime}} h\left(s, t^{\prime}\right)$ for all stacks $s$ and periods $t$. With these speedups, the new worst-case complexity of the algorithm is thus $O\left(M \times W \times H_{\max }\right)$. 


\section{Numerical experiments}

The proposed local search algorithm (LS) has been implemented in C++. All tests have been carried out on Intel Xeon E5-2650 v2 CPUs (25M Cache, $2.60 \mathrm{GHz}$ ). Each computing node has 2 processors with 8 cores each and 64 GB RAM. In total, up to 5 jobs run in parallel on one node, sharing memory.

In order to analyze its performance, we apply our algorithm to three sets of instances. Two of which have been proposed by other authors (Caserta et al. (2011) and Tricoire et al. (2018)) while the third set is new. The small instances of Caserta et al. (2011) and the large instances proposed by Tricoire et al. (2018) follow the same scheme. Let $H$ denote the maximum height of a stack in an initial configuration, the size of the Caserta et al. instances ranges from $(H, W)=(3,3)$ until $(H, W)=(10,10)$, the size of the Tricoire et al. instances ranges from $(H, W)=(10,10)$ until $(H, W)=(100,100)$, where we recall that $W$ is the number of stacks. Furthermore, like in previous works, we consider two different stack height limitations for each data set: $H_{\max }=H+2$ and $H_{\max }=$ unlimited. Each instance class defined by $(H, W)$ contains 40 instances.

The purpose of the new set of wide instances is to mimic the actual height limitations of stacker cranes at container yards. Therefore, we set $H=10$ and we only increase the width $W$. We start with $(H, W)=(10,10)$ and we increase it in steps of 10 until $(H, W)=$ $(10,100)$. As previously, we consider two stack height limitations, namely $H_{\max }=H+2$ and $H_{\max }=$ unlimited, and we generate 40 instances for each instance class.

Since the aim of our LS is to improve on a previously generated solution, we apply it to starting solutions produced by different constructive heuristics. For the small Caserta instances, we use the rake search and pilot method of Tricoire et al. (2018) as well as two versions of the greedy look ahead heuristic (GLAH) of Jin et al. (2015). For all other instances, we use the following four fast construction heuristics:

- JZW: evaluation subroutine of the greedy look ahead heuristic of Jin et al. (2015). This subroutine considers several different conditions for identifying the destination stack of a container which needs to be relocated, including the simultaneous relocation of another container if this seems advantageous.

- LA-S-1: extended look-ahead heuristic of Petering and Hussein (2013), where "S-1" defines the look ahead in terms of containers and here it is equal to $W-1$. It involves cleaning moves. A cleaning move is a relocation of a container, whose relocation is not mandatory, to another stack from where it does not have to be relocated again.

- SM-2: heuristic incorporating best safe 1-relocate moves and best safe 2-relocate moves (Tricoire et al., 2018). Safe relocations are those which do not increase the proposed lower bound on the number of necessary relocations.

- SmSEQ-2: combination of decreasing sequence relocate (sequence length $\geq 1$ ) and SM-2 (Tricoire et al., 2018). Decreasing sequences are sequences of containers which are stacked in the opposite order compared to the sequence in which they need to be retrieved. In certain cases, it is beneficial to relocate the whole sequence to some other stack $s$, even if that involves making room for it in $s$ first. 
For all construction methods, we use the implementation from Tricoire et al. (2018).

Many of the small instances from Caserta et al. (2011) are solved optimally with constructive methods and the necessity of developing complex local search operators is not obvious for these instances. Even so, we evaluated our approach on these instances and obtained only very limited improvements. These experiments are reported in Tables 4 and 5 in the appendix. More interesting results are obtained with larger instances like the ones introduced in Tricoire et al. (2018).

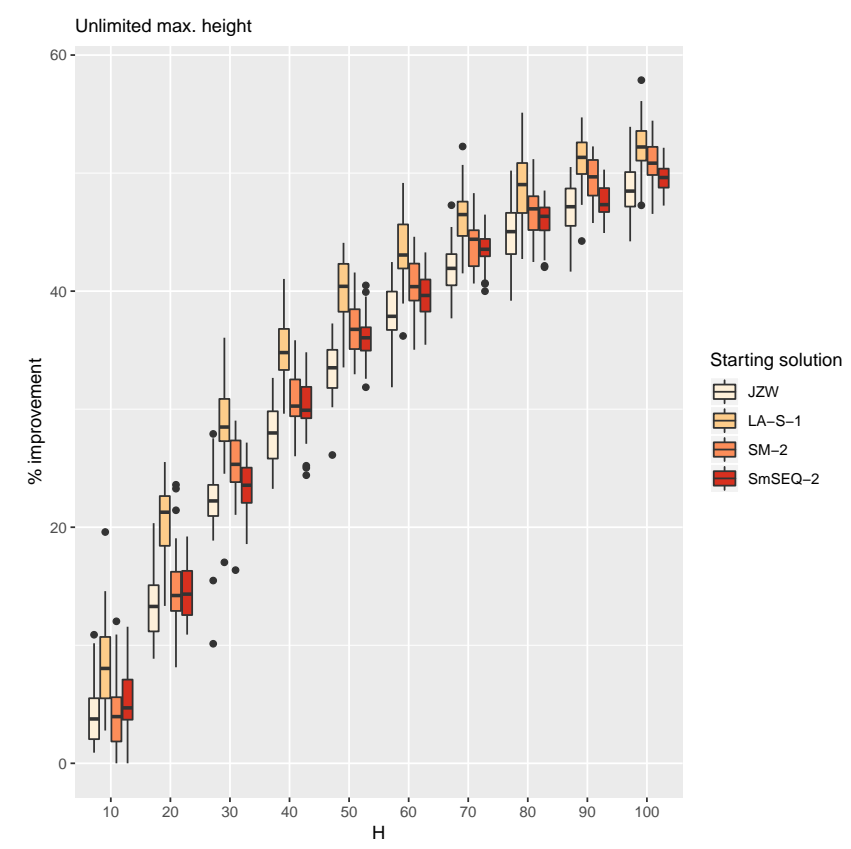

Figure 5: Average percentage improvement compared to starting solution for benchmark instances of Tricoire et al. (2018) with $H_{\max }=$ unlimited and $H \in$ $\{10,20,30,40,50,60,70,80,90,100\}$

In a first stage, we analyze the improvement potential of our local search algorithm. For this purpose, we plot the percentage improvement (across 40 instances) per instance class using the respective objective value obtained from the constructive heuristic as a base line. Figures 5-8 provide the obtained results for $H_{\max }=$ unlimited and $H_{\max }=H+2$, respectively. For the instances of Tricoire et al. (2018) an obvious trend can be observed: the larger the instance, the larger the improvement by our local search algorithm. The improvements are consistently above $40 \%$ for the largest instances without height limitation and above $20 \%$ with $H_{\max }=H+2$. The respective average solution values per instance class, along with the average improvement compared to the starting solution, are given in Tables 8 and 9 in the appendix.

For the wide instances the picture is different. We find improvements (detailed results can be found in Tables 10 and 11 in the Appendix) but they are much less pronounced than for the large instances. Obviously, the larger the width of the instance the smaller the improvement potential.

In a second stage, we consider for any given instance $x$ two values: 


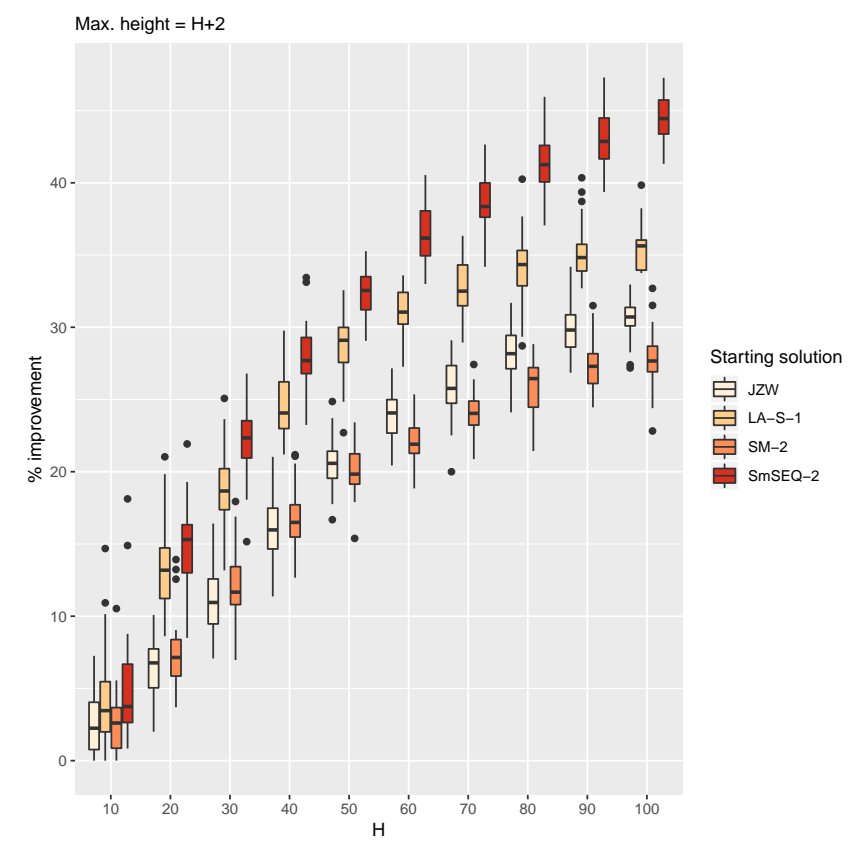

Figure 6: Average percentage improvement compared to starting solution for benchmark instances of Tricoire et al. (2018) with $H_{\max }=H+2$ and $H \in$ $\{10,20,30,40,50,60,70,80,90,100\}$

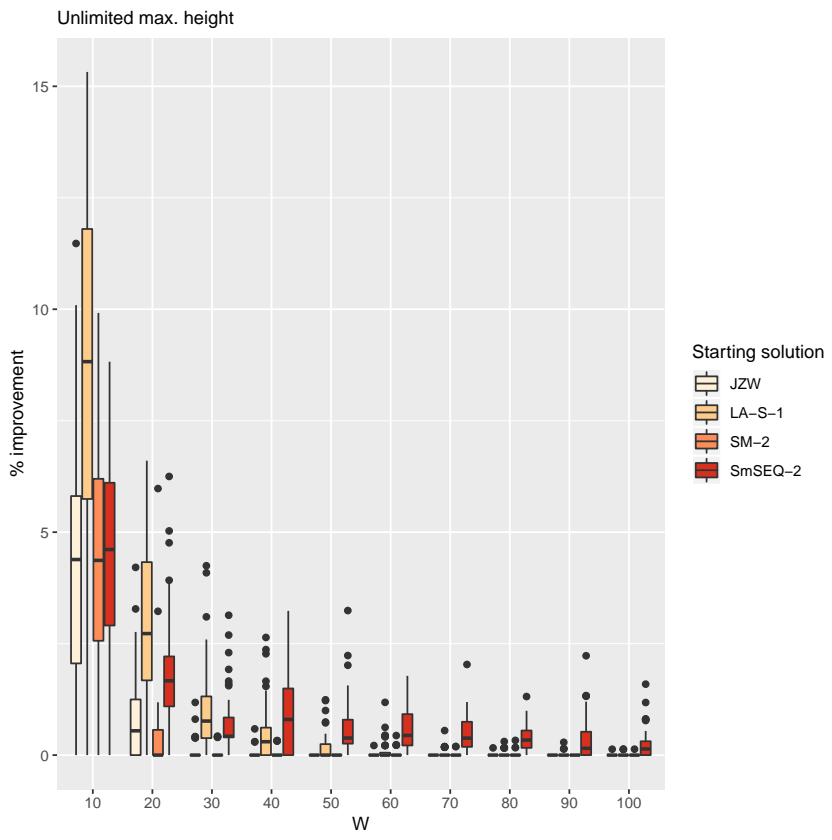

Figure 7: Average percentage improvement compared to starting solution for wide instances with $H_{\max }=$ unlimited and $W \in\{10,20,30,40,50,60,70,80,90,100\}$ 


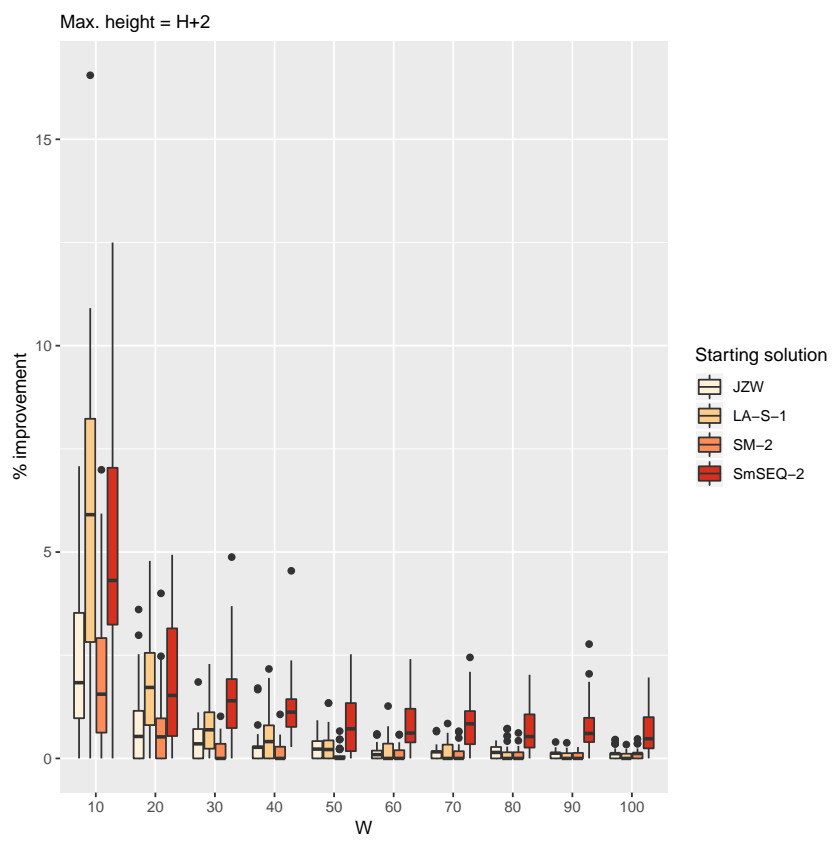

Figure 8: Average percentage improvement compared to starting solution for wide instances with $H_{\max }=H+2$ and $W \in\{10,20,30,40,50,60,70,80,90,100\}$

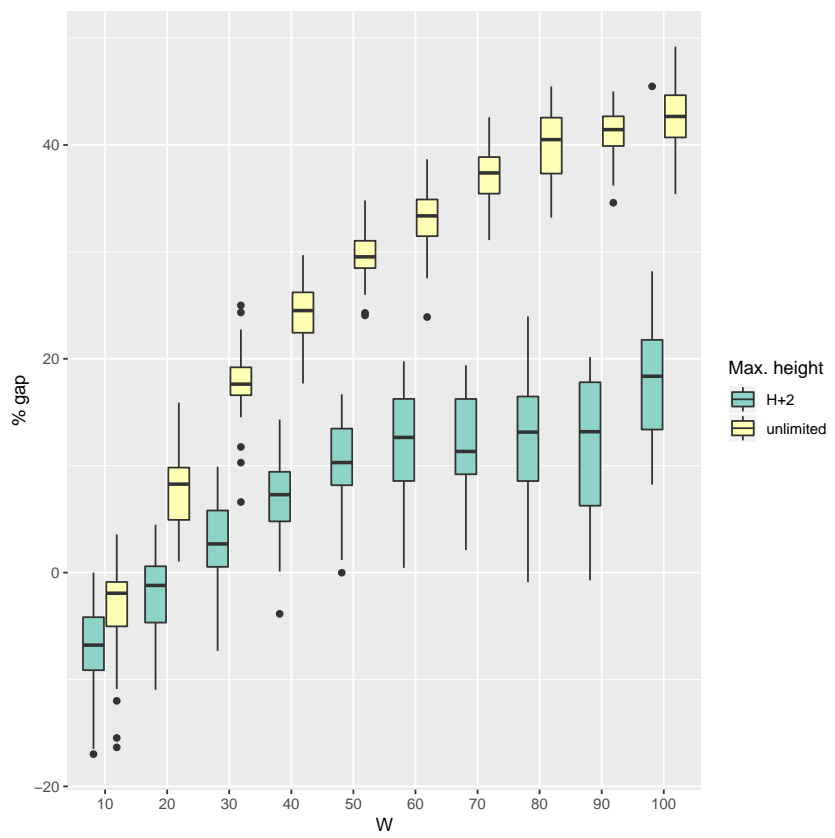

Figure 9: Worst percentage improvement compared to best constructive solution across all methods for benchmark instances of Tricoire et al. (2018) 


\begin{tabular}{cccccc}
\hline$H$ & $W$ & LA-S-1 & SM-2 & SmSEQ-2 & JZW \\
\hline 10 & 10 & 0.00 & 0.00 & 0.00 & 0.00 \\
20 & 20 & 0.53 & 0.39 & 0.34 & 0.35 \\
30 & 30 & 7.73 & 6.14 & 3.97 & 5.01 \\
40 & 40 & 63.60 & 47.89 & 30.40 & 40.47 \\
50 & 50 & 323.99 & 254.74 & 162.90 & 219.89 \\
60 & 60 & 1210.38 & 1000.28 & 595.52 & 910.88 \\
70 & 70 & 3630.31 & 3101.77 & 1935.90 & 2836.54 \\
80 & 80 & 8398.51 & 7196.79 & 4524.30 & 6536.57 \\
90 & 90 & 18966.91 & 16862.56 & 10333.31 & 15286.99 \\
100 & 100 & 38592.69 & 34182.83 & 21072.38 & 31421.58 \\
\hline
\end{tabular}

Table 2: CPU time in seconds of LS, using different methods for the starting solution. Tricoire instances, $H_{\max }=$ unlimited.

\begin{tabular}{cccccc}
\hline$H$ & $W$ & LA-S-1 & SM-2 & SmSEQ-2 & JZW \\
\hline 10 & 10 & 0.00 & 0.00 & 0.00 & 0.00 \\
20 & 20 & 0.75 & 0.55 & 0.49 & 0.49 \\
30 & 30 & 12.74 & 8.47 & 7.00 & 7.33 \\
40 & 40 & 123.62 & 87.48 & 59.19 & 71.14 \\
50 & 50 & 769.24 & 515.80 & 329.88 & 434.28 \\
60 & 60 & 3579.65 & 2618.78 & 1509.08 & 2141.47 \\
70 & 70 & 13303.65 & 9031.78 & 4933.01 & 7534.28 \\
80 & 80 & 39538.48 & 27273.29 & 13638.50 & 21612.92 \\
90 & 90 & 113666.67 & 78958.13 & 35068.10 & 61040.70 \\
100 & 100 & $237755.00(31)$ & $192664.73(7)$ & 85194.16 & $154284.41(3)$ \\
\hline
\end{tabular}

Table 3: CPU time in seconds of LS, using different methods for the starting solution. Tricoire instances, $H_{\max }=H+2$. The number of instances which could not be solved due to time restrictions is mentioned in parentheses.

- $B B(x)$ is the best solution value for instance $x$ among all starting solutions, i.e. before LS

- $W A(x)$ is the worst solution value for instance $x$ among all post-LS solutions, i.e. after LS.

In order to evaluate the overall performance of our LS, we compare the $B B(x)$ against $W A(x)$ instance-wise. This comparison (again in terms of percentage deviations) is given in Figure 9 . It illustrates that, in the case of the benchmark instances of Tricoire et al. (2018) except for the smallest instances, the worst solution obtained by LS is usually better than the best solution identified with the best performing constructive method. This improvement is more pronounced for instances without a height restriction, i.e. $H_{\max }=$ unlimited: more than $30 \%$ for instances with $H$ and $W$ both $\geq 70$. This emphasizes the value of the proposed local search algorithm: on these instances, as long as LS is applied, it does not matter which construction method is used to begin with, the end result will almost always be better than what any construction method could do.

Information on the required CPU time in seconds for the instances of Tricoire et al. (2018) can be found in Tables 2 and 3 . Each entry gives the average CPU time across 40 instances per instance class and in parentheses the number of instances which could not be solved due to CPU budget limitation. Quite obviously, CPU times increase with instance size, sometimes exceeding the time limit for the largest instances with $H_{\max }=H+2$. However, we note that for the largest instances the average obtained improvement amounts to at least $20 \%$ for SM-2 
and to more than $40 \%$ when applied to solutions obtained by SmSEQ-2. We also note that these values are due to running the full fledged LS until termination. Truncated versions with a reduced state-space or a CPU time limit may equally be envisaged.

For all other instances, the required CPU effort was systematically less than a second; therefore we do not report it.

\section{Conclusions and perspectives}

We have introduced the first local search based heuristic algorithm for the unrestricted block relocation problem; it relies on dynamic programming and we were able to illustrate its value in extensive tests on large benchmark instances, reaching improvements of up to 50\% compared to starting solutions. These starting solutions are obtained by state-of-the-art constructive heuristics. Our results nicely show the unexpectedly high potential for improvement on these large BRP instances.

Future research will involve the design of more efficient improvement methods, so as to obtain solutions of comparable quality with lower time requirements. Apart from cases when the aspiration speedup is triggered, the local search aims at finding the best relocation sequence; it potentially could be accelerated by limiting the search to any improving sequence or by voluntarily limiting the number of states explored. In addition, the local search operator could be integrated into a metaheuristic scheme, instead of being used in a simple greedy descent algorithm.

Another perspective of our work is the design of more complex local search schemes which do not only consider a single container at a time, but two or more. Our results for the wide instances indicate the need for such more complex operators.

Extensions of the dynamic programming scheme to other variants of the BRP will also be investigated. A natural extension is to the restricted BRP. Less transitions would be accepted, which would reduce the state-space and accelerate the algorithm, but it would also limit the opportunities for improvement. Problems in which the priority order is defined among groups of containers could also be considered, with the opposite effect: the state-space would increase a lot, so as the chances of improvement.

\section{Acknowledgments}

The authors thank the referees for their acute comments, which significantly contributed to enhance the quality of this paper.

\section{References}

Azari, E., Eskandari, H., and Nourmohammadi, A. (2017). Decreasing the crane working time in retrieving the containers from a bay. Scientia Iranica. Transaction E, Industrial Engineering, 24(1):309. 
Caserta, M., Schwarze, S., and Voß, S. (2012). A mathematical formulation and complexity considerations for the blocks relocation problem. European Journal of Operational Research, 219(1):96-104.

Caserta, M., Voß, S., and Sniedovich, M. (2011). Applying the corridor method to a blocks relocation problem. OR spectrum, 33(4):915-929.

de Melo da Silva, M., Toulouse, S., and Calvo, R. W. (2018). A new effective unified model for solving the pre-marshalling and block relocation problems. European Journal of Operational Research, 271(1):40 - 56 .

Eskandari, H. and Azari, E. (2015). Notes on mathematical formulation and complexity considerations for blocks relocation problem. Scientia Iranica. Transaction E, Industrial Engineering, 22(6):2722.

Expósito-Izquierdo, C., Melián-Batista, B., and Moreno-Vega, J. M. (2015). An exact approach for the blocks relocation problem. Expert Systems with Applications, 42(17-18):64086422 .

Forster, F. and Bortfeldt, A. (2012). A tree search procedure for the container relocation problem. Computers \& Operations Research, 39(2):299-309.

Galle, V., Barnhart, C., and Jaillet, P. (2018). A new binary formulation of the restricted container relocation problem based on a binary encoding of configurations. European Journal of Operational Research, 267:467-477.

Hakan Akyüz, M. and Lee, C.-Y. (2014). A mathematical formulation and efficient heuristics for the dynamic container relocation problem. Naval Research Logistics (NRL), 61(2):101118.

Jin, B., Zhu, W., and Lim, A. (2015). Solving the container relocation problem by an improved greedy look-ahead heuristic. European Journal of Operational Research, 240(3):837-847.

Jovanovic, R., Tuba, M., and Voß, S. (2017). A multi-heuristic approach for solving the pre-marshalling problem. Central European Journal of Operations Research, 25(1):1-28.

Jovanovic, R. and Voß, S. (2014). A chain heuristic for the blocks relocation problem. Computers \& Industrial Engineering, 75:79-86.

Kim, K. H. and Hong, G.-P. (2006). A heuristic rule for relocating blocks. Computers \& Operations Research, 33(4):940-954.

$\mathrm{Ku}$, D. and Arthanari, T. S. (2016a). Container relocation problem with time windows for container departure. European Journal of Operational Research, 252(3):1031-1039.

$\mathrm{Ku}$, D. and Arthanari, T. S. (2016b). On the abstraction method for the container relocation problem. Computers $\&$ Operations Research, 68:110-122.

Lehnfeld, J. and Knust, S. (2014). Loading, unloading and premarshalling of stacks in storage areas: Survey and classification. European Journal of Operational Research, 239(2):297312 . 
Parreño Torres, C. and Alvarez-Valdes, R. (2019). Integer programming models for the premarshalling problem. European Journal of Operational Research, 274:142-154.

Petering, M. E. and Hussein, M. I. (2013). A new mixed integer program and extended lookahead heuristic algorithm for the block relocation problem. European Journal of Operational Research, 231(1):120-130.

Prandtstetter, M. (2013). A dynamic programming based branch-and-bound algorithm for the container pre-marshalling problem. Technical report, Technical report, AIT Austrian Institute of Technology.

Quispe, K., Lintzmayer, C., and Xavier, E. (2018). An exact algorithm for the blocks relocation problem with new lower bounds. Computers and Operations Research, 99:206-217.

Tanaka, S. and Mizuno, F. (2015). Dominance properties for the unrestricted block relocation problem and their application to a branch-and-bound algorithm. In Automation Science and Engineering (CASE), 2015 IEEE International Conference on, pages 509-514. IEEE.

Tanaka, S. and Mizuno, F. (2018). An exact algorithm for the unrestricted block relocation problem. Computers \& Operations Research, 95:12-31.

Tanaka, S. and Tierney, K. (2018). Solving real-world sized container pre-marshalling problems with an iterative deepening branch-and-bound algorithm. European Journal of Operational Research, 264:165-180.

Tricoire, F., Scagnetti, J., and Beham, A. (2018). New insights on the block relocation problem. Computers $\&$ Operations Research, 89:127-139.

Zhu, W., Qin, H., Lim, A., and Zhang, H. (2012). Iterative deepening a* algorithms for the container relocation problem. IEEE Transactions on Automation Science and Engineering, $9(4): 710-722$.

\section{A Results for Caserta et al. instances}

Tables 4,7 provide the following information for each instance class from Caserta et al. (2011): the average improvement (Avg. impr.) obtained by means of LS, the number of instances for which LS found an improved solution (\# impr.) and the average CPU effort of LS in seconds. Average improvement represents the average number of relocations saved by applying local search. These indicators are reported for each of the four advanced methods (rake search and pilot method of Tricoire et al. (2018), two versions of the greedy look ahead heuristic (GLAH) of Jin et al. (2015)) and for the four fast constructive methods (LA-S-1, SM-2, SmSEQ-2, JZW). Each row represents 40 instances.

\section{B Detailed results for Tricoire instances}

Tables 8 and 9 provide average values across 40 instances per instance class of the instances from Tricoire et al. (2018). The starting solutions are obtained by means of the fast constructive methods, then LS is applied. Average values before and after LS are reported; in 


\begin{tabular}{cccccccccccccc}
\hline & \multicolumn{4}{c}{ Rake search } & \multicolumn{4}{c}{ Pilot method } & \multicolumn{3}{c}{ GLAH-3 } & \multicolumn{3}{c}{ GLAH-4 } \\
$H$ & Wvg. & $\begin{array}{c}\text { Avg. } \\
\text { impr. }\end{array}$ & $\begin{array}{c}\# \\
\text { impr. }\end{array}$ & CPU & $\begin{array}{c}\text { Avpr. } \\
\text { impr. }\end{array}$ & $\begin{array}{c}\text { Avg. } \\
\text { impr. }\end{array}$ & CPU & impr. & impr. & CPU & $\begin{array}{c}\text { Avg. } \\
\text { impr. }\end{array}$ & $\begin{array}{c}\# \\
\text { impr. }\end{array}$ & CPU \\
\hline 3 & 3 & 0.00 & 0 & 0.00 & 0.00 & 0 & 0.00 & 0.00 & 0 & 0.00 & 0.00 & 0 & 0.00 \\
3 & 4 & 0.00 & 0 & 0.00 & 0.00 & 0 & 0.00 & 0.00 & 0 & 0.00 & 0.00 & 0 & 0.00 \\
3 & 5 & 0.00 & 0 & 0.00 & 0.00 & 0 & 0.00 & 0.00 & 0 & 0.00 & 0.00 & 0 & 0.00 \\
3 & 6 & 0.00 & 0 & 0.00 & 0.00 & 0 & 0.00 & 0.00 & 0 & 0.00 & 0.00 & 0 & 0.00 \\
3 & 7 & 0.00 & 0 & 0.00 & 0.00 & 0 & 0.00 & 0.00 & 0 & 0.00 & 0.00 & 0 & 0.00 \\
3 & 8 & 0.00 & 0 & 0.00 & 0.00 & 0 & 0.00 & 0.00 & 0 & 0.00 & 0.00 & 0 & 0.00 \\
4 & 4 & 0.00 & 0 & 0.00 & 0.00 & 0 & 0.00 & 0.00 & 0 & 0.00 & 0.00 & 0 & 0.00 \\
4 & 5 & 0.00 & 0 & 0.00 & 0.00 & 0 & 0.00 & 0.03 & 1 & 0.00 & 0.03 & 1 & 0.00 \\
4 & 6 & 0.00 & 0 & 0.00 & 0.00 & 0 & 0.00 & 0.00 & 0 & 0.00 & 0.00 & 0 & 0.00 \\
4 & 7 & 0.00 & 0 & 0.00 & 0.00 & 0 & 0.00 & 0.00 & 0 & 0.00 & 0.00 & 0 & 0.00 \\
5 & 4 & 0.00 & 0 & 0.00 & 0.03 & 1 & 0.00 & 0.00 & 0 & 0.00 & 0.00 & 0 & 0.00 \\
5 & 5 & 0.00 & 0 & 0.00 & 0.03 & 1 & 0.00 & 0.00 & 0 & 0.00 & 0.00 & 0 & 0.00 \\
5 & 6 & 0.00 & 0 & 0.00 & 0.00 & 0 & 0.00 & 0.00 & 0 & 0.00 & 0.00 & 0 & 0.00 \\
5 & 7 & 0.00 & 0 & 0.00 & 0.00 & 0 & 0.00 & 0.03 & 1 & 0.00 & 0.00 & 0 & 0.00 \\
5 & 8 & 0.00 & 0 & 0.00 & 0.00 & 0 & 0.00 & 0.00 & 0 & 0.00 & 0.00 & 0 & 0.00 \\
5 & 9 & 0.00 & 0 & 0.00 & 0.03 & 1 & 0.00 & 0.00 & 0 & 0.00 & 0.00 & 0 & 0.00 \\
5 & 10 & 0.03 & 1 & 0.00 & 0.00 & 0 & 0.00 & 0.00 & 0 & 0.00 & 0.00 & 0 & 0.00 \\
6 & 6 & 0.07 & 3 & 0.00 & 0.07 & 3 & 0.00 & 0.00 & 0 & 0.00 & 0.00 & 0 & 0.00 \\
6 & 10 & 0.03 & 1 & 0.00 & 0.03 & 1 & 0.00 & 0.00 & 0 & 0.00 & 0.03 & 1 & 0.00 \\
10 & 6 & 2.58 & 35 & 0.00 & 1.80 & 30 & 0.00 & 1.15 & 24 & 0.00 & 1.27 & 29 & 0.00 \\
10 & 10 & 1.55 & 29 & 0.00 & 0.72 & 20 & 0.00 & 0.90 & 21 & 0.00 & 0.82 & 18 & 0.00 \\
\hline
\end{tabular}

Table 4: Improvement by LS, using different methods for the starting solution. Caserta instances, $H_{\max }=$ unlimited.

\begin{tabular}{|c|c|c|c|c|c|c|c|c|c|c|c|c|c|}
\hline \multirow[b]{2}{*}{$H$} & \multirow[b]{2}{*}{$W$} & \multicolumn{3}{|c|}{ Rake search } & \multicolumn{3}{|c|}{ Pilot method } & \multicolumn{3}{|c|}{ GLAH-3 } & \multicolumn{3}{|c|}{ GLAH-4 } \\
\hline & & $\begin{array}{l}\text { Avg. } \\
\text { impr. }\end{array}$ & $\begin{array}{c}\# \\
\text { impr. }\end{array}$ & $\mathrm{CPU}$ & $\begin{array}{l}\text { Avg. } \\
\text { impr. }\end{array}$ & $\begin{array}{c}\# \\
\text { impr. }\end{array}$ & $\mathrm{CPU}$ & $\begin{array}{l}\text { Avg. } \\
\text { impr. }\end{array}$ & $\begin{array}{c}\# \\
\text { impr. }\end{array}$ & $\mathrm{CPU}$ & $\begin{array}{l}\text { Avg. } \\
\text { impr. }\end{array}$ & $\begin{array}{c}\# \\
\text { impr. }\end{array}$ & $\mathrm{CPU}$ \\
\hline 3 & 3 & 0.00 & 0 & 0.00 & 0.00 & 0 & 0.00 & 0.00 & 0 & 0.00 & 0.00 & 0 & 0.00 \\
\hline 3 & 4 & 0.00 & 0 & 0.00 & 0.00 & 0 & 0.00 & 0.00 & 0 & 0.00 & 0.00 & 0 & 0.00 \\
\hline 3 & 5 & 0.00 & 0 & 0.00 & 0.00 & 0 & 0.00 & 0.00 & 0 & 0.00 & 0.00 & 0 & 0.00 \\
\hline 3 & 6 & 0.00 & 0 & 0.00 & 0.00 & 0 & 0.00 & 0.00 & 0 & 0.00 & 0.00 & 0 & 0.00 \\
\hline 3 & 7 & 0.00 & 0 & 0.00 & 0.00 & 0 & 0.00 & 0.00 & 0 & 0.00 & 0.00 & 0 & 0.00 \\
\hline 3 & 8 & 0.00 & 0 & 0.00 & 0.00 & 0 & 0.00 & 0.00 & 0 & 0.00 & 0.00 & 0 & 0.00 \\
\hline 4 & 4 & 0.00 & 0 & 0.00 & 0.00 & 0 & 0.00 & 0.00 & 0 & 0.00 & 0.00 & 0 & 0.00 \\
\hline 4 & 5 & 0.00 & 0 & 0.00 & 0.00 & 0 & 0.00 & 0.03 & 1 & 0.00 & 0.03 & 1 & 0.00 \\
\hline 4 & 6 & 0.00 & 0 & 0.00 & 0.00 & 0 & 0.00 & 0.00 & 0 & 0.00 & 0.00 & 0 & 0.00 \\
\hline 4 & 7 & 0.00 & 0 & 0.00 & 0.00 & 0 & 0.00 & 0.00 & 0 & 0.00 & 0.00 & 0 & 0.00 \\
\hline 5 & 4 & 0.03 & 1 & 0.00 & 0.03 & 1 & 0.00 & 0.05 & 2 & 0.00 & 0.03 & 1 & 0.00 \\
\hline 5 & 5 & 0.00 & 0 & 0.00 & 0.03 & 1 & 0.00 & 0.03 & 1 & 0.00 & 0.00 & 0 & 0.00 \\
\hline 5 & 6 & 0.00 & 0 & 0.00 & 0.00 & 0 & 0.00 & 0.00 & 0 & 0.00 & 0.00 & 0 & 0.00 \\
\hline 5 & 7 & 0.03 & 1 & 0.00 & 0.00 & 0 & 0.00 & 0.03 & 1 & 0.00 & 0.03 & 1 & 0.00 \\
\hline 5 & 8 & 0.00 & 0 & 0.00 & 0.00 & 0 & 0.00 & 0.03 & 1 & 0.00 & 0.00 & 0 & 0.00 \\
\hline 5 & 9 & 0.03 & 1 & 0.00 & 0.03 & 1 & 0.00 & 0.00 & 0 & 0.00 & 0.00 & 0 & 0.00 \\
\hline 5 & 10 & 0.00 & 0 & 0.00 & 0.00 & 0 & 0.00 & 0.00 & 0 & 0.00 & 0.00 & 0 & 0.00 \\
\hline 6 & 6 & 0.07 & 2 & 0.00 & 0.05 & 2 & 0.00 & 0.00 & 0 & 0.00 & 0.00 & 0 & 0.00 \\
\hline 6 & 10 & 0.05 & 2 & 0.00 & 0.03 & 1 & 0.00 & 0.00 & 0 & 0.00 & 0.00 & 0 & 0.00 \\
\hline 10 & 6 & 1.50 & 25 & 0.00 & 0.95 & 20 & 0.00 & 0.88 & 17 & 0.00 & 0.88 & 21 & 0.00 \\
\hline 10 & 10 & 1.00 & 21 & 0.00 & 0.47 & 16 & 0.00 & 0.53 & 13 & 0.00 & 0.35 & 11 & 0.00 \\
\hline
\end{tabular}

Table 5: Improvement by LS, using different methods for the starting solution. Caserta instances, $H_{\max }=H+2$. 


\begin{tabular}{cccccccccccccc}
\hline & & \multicolumn{3}{c}{ LA-S-1 } & \multicolumn{3}{c}{ SM-2 } & \multicolumn{3}{c}{ SmSEQ-2 } & \multicolumn{3}{c}{ JZW } \\
$H$ & $W$ & $\begin{array}{c}\text { Avg. } \\
\text { impr. }\end{array}$ & $\begin{array}{c}\# \\
\text { impr. }\end{array}$ & CPU & $\begin{array}{c}\text { Avg. } \\
\text { impr. }\end{array}$ & $\begin{array}{c}\# \\
\text { impr. }\end{array}$ & CPU & $\begin{array}{c}\text { Avg. } \\
\text { impr. }\end{array}$ & $\begin{array}{c}\text { Avg. } \\
\text { impr. }\end{array}$ & CPU & $\begin{array}{c}\text { Avpr. } \\
\text { impr. }\end{array}$ & CPU \\
\hline 3 & 3 & 0.03 & 1 & 0.00 & 0.00 & 0 & 0.00 & 0.00 & 0 & 0.00 & 0.03 & 1 & 0.00 \\
3 & 4 & 0.00 & 0 & 0.00 & 0.00 & 0 & 0.00 & 0.00 & 0 & 0.00 & 0.00 & 0 & 0.00 \\
3 & 5 & 0.00 & 0 & 0.00 & 0.00 & 0 & 0.00 & 0.00 & 0 & 0.00 & 0.00 & 0 & 0.00 \\
3 & 6 & 0.00 & 0 & 0.00 & 0.00 & 0 & 0.00 & 0.00 & 0 & 0.00 & 0.00 & 0 & 0.00 \\
3 & 7 & 0.00 & 0 & 0.00 & 0.00 & 0 & 0.00 & 0.00 & 0 & 0.00 & 0.00 & 0 & 0.00 \\
3 & 8 & 0.00 & 0 & 0.00 & 0.00 & 0 & 0.00 & 0.00 & 0 & 0.00 & 0.00 & 0 & 0.00 \\
4 & 4 & 0.25 & 8 & 0.00 & 0.07 & 3 & 0.00 & 0.12 & 4 & 0.00 & 0.03 & 1 & 0.00 \\
4 & 5 & 0.07 & 3 & 0.00 & 0.00 & 0 & 0.00 & 0.05 & 2 & 0.00 & 0.03 & 1 & 0.00 \\
4 & 6 & 0.07 & 2 & 0.00 & 0.00 & 0 & 0.00 & 0.03 & 1 & 0.00 & 0.07 & 3 & 0.00 \\
4 & 7 & 0.03 & 1 & 0.00 & 0.03 & 1 & 0.00 & 0.03 & 1 & 0.00 & 0.03 & 1 & 0.00 \\
5 & 4 & 0.45 & 14 & 0.00 & 0.33 & 7 & 0.00 & 0.30 & 10 & 0.00 & 0.20 & 7 & 0.00 \\
5 & 5 & 0.57 & 20 & 0.00 & 0.23 & 6 & 0.00 & 0.53 & 15 & 0.00 & 0.35 & 13 & 0.00 \\
5 & 6 & 0.25 & 8 & 0.00 & 0.05 & 2 & 0.00 & 0.17 & 5 & 0.00 & 0.12 & 5 & 0.00 \\
5 & 7 & 0.30 & 7 & 0.00 & 0.00 & 0 & 0.00 & 0.20 & 6 & 0.00 & 0.17 & 7 & 0.00 \\
5 & 8 & 0.17 & 5 & 0.00 & 0.00 & 0 & 0.00 & 0.05 & 2 & 0.00 & 0.05 & 1 & 0.00 \\
5 & 9 & 0.12 & 5 & 0.00 & 0.00 & 0 & 0.00 & 0.17 & 5 & 0.00 & 0.07 & 2 & 0.00 \\
5 & 10 & 0.05 & 2 & 0.00 & 0.00 & 0 & 0.00 & 0.15 & 5 & 0.00 & 0.05 & 2 & 0.00 \\
6 & 6 & 1.23 & 22 & 0.00 & 0.53 & 15 & 0.00 & 0.80 & 19 & 0.00 & 0.50 & 15 & 0.00 \\
6 & 10 & 0.42 & 11 & 0.00 & 0.05 & 1 & 0.00 & 0.28 & 6 & 0.00 & 0.20 & 7 & 0.00 \\
10 & 6 & 10.22 & 40 & 0.00 & 8.00 & 40 & 0.00 & 9.07 & 40 & 0.00 & 6.80 & 39 & 0.00 \\
10 & 10 & 11.65 & 39 & 0.00 & 4.20 & 37 & 0.00 & 6.20 & 39 & 0.00 & 3.90 & 39 & 0.00 \\
\hline
\end{tabular}

Table 6: Improvement by LS, using different methods for the starting solution. Caserta instances, $H_{\max }=$ unlimited.

\begin{tabular}{|c|c|c|c|c|c|c|c|c|c|c|c|c|c|}
\hline \multirow[b]{2}{*}{$H$} & \multirow[b]{2}{*}{$W$} & \multicolumn{3}{|c|}{ LA-S-1 } & \multicolumn{3}{|c|}{ SM-2 } & \multicolumn{3}{|c|}{ SmSEQ-2 } & \multicolumn{3}{|c|}{ JZW } \\
\hline & & $\begin{array}{l}\text { Avg. } \\
\text { impr. }\end{array}$ & $\begin{array}{c}\# \\
\text { impr. }\end{array}$ & $\mathrm{CPU}$ & $\begin{array}{l}\text { Avg. } \\
\text { impr. }\end{array}$ & $\begin{array}{c}\# \\
\text { impr. }\end{array}$ & $\mathrm{CPU}$ & $\begin{array}{l}\text { Avg. } \\
\text { impr. }\end{array}$ & $\begin{array}{c}\# \\
\text { impr. }\end{array}$ & $\mathrm{CPU}$ & $\begin{array}{l}\text { Avg. } \\
\text { impr. }\end{array}$ & $\begin{array}{c}\# \\
\text { impr. }\end{array}$ & $\mathrm{CPU}$ \\
\hline 3 & 3 & 0.03 & 1 & 0.00 & 0.00 & 0 & 0.00 & 0.00 & 0 & 0.00 & 0.03 & 1 & 0.00 \\
\hline 3 & 4 & 0.00 & 0 & 0.00 & 0.00 & 0 & 0.00 & 0.00 & 0 & 0.00 & 0.00 & 0 & 0.00 \\
\hline 3 & 5 & 0.00 & 0 & 0.00 & 0.00 & 0 & 0.00 & 0.00 & 0 & 0.00 & 0.00 & 0 & 0.00 \\
\hline 3 & 6 & 0.00 & 0 & 0.00 & 0.00 & 0 & 0.00 & 0.00 & 0 & 0.00 & 0.00 & 0 & 0.00 \\
\hline 3 & 7 & 0.00 & 0 & 0.00 & 0.00 & 0 & 0.00 & 0.00 & 0 & 0.00 & 0.00 & 0 & 0.00 \\
\hline 3 & 8 & 0.00 & 0 & 0.00 & 0.00 & 0 & 0.00 & 0.00 & 0 & 0.00 & 0.00 & 0 & 0.00 \\
\hline 4 & 4 & 0.20 & 7 & 0.00 & 0.00 & 0 & 0.00 & 0.05 & 2 & 0.00 & 0.05 & 2 & 0.00 \\
\hline 4 & 5 & 0.10 & 4 & 0.00 & 0.00 & 0 & 0.00 & 0.07 & 3 & 0.00 & 0.05 & 2 & 0.00 \\
\hline 4 & 6 & 0.10 & 3 & 0.00 & 0.00 & 0 & 0.00 & 0.03 & 1 & 0.00 & 0.05 & 2 & 0.00 \\
\hline 4 & 7 & 0.03 & 1 & 0.00 & 0.00 & 0 & 0.00 & 0.03 & 1 & 0.00 & 0.07 & 3 & 0.00 \\
\hline 5 & 4 & 0.40 & 11 & 0.00 & 0.20 & 7 & 0.00 & 0.33 & 9 & 0.00 & 0.28 & 9 & 0.00 \\
\hline 5 & 5 & 0.53 & 14 & 0.00 & 0.07 & 3 & 0.00 & 0.45 & 11 & 0.00 & 0.15 & 6 & 0.00 \\
\hline 5 & 6 & 0.12 & 4 & 0.00 & 0.07 & 2 & 0.00 & 0.17 & 5 & 0.00 & 0.12 & 5 & 0.00 \\
\hline 5 & 7 & 0.17 & 5 & 0.00 & 0.00 & 0 & 0.00 & 0.23 & 6 & 0.00 & 0.15 & 6 & 0.00 \\
\hline 5 & 8 & 0.12 & 4 & 0.00 & 0.03 & 1 & 0.00 & 0.07 & 3 & 0.00 & 0.12 & 4 & 0.00 \\
\hline 5 & 9 & 0.15 & 5 & 0.00 & 0.07 & 2 & 0.00 & 0.25 & 10 & 0.00 & 0.07 & 3 & 0.00 \\
\hline 5 & 10 & 0.03 & 1 & 0.00 & 0.03 & 1 & 0.00 & 0.17 & 5 & 0.00 & 0.10 & 4 & 0.00 \\
\hline 6 & 6 & 0.90 & 23 & 0.00 & 0.30 & 11 & 0.00 & 0.88 & 18 & 0.00 & 0.55 & 15 & 0.00 \\
\hline 6 & 10 & 0.35 & 10 & 0.00 & 0.03 & 1 & 0.00 & 0.30 & 8 & 0.00 & 0.25 & 8 & 0.00 \\
\hline 10 & 6 & 7.17 & 39 & 0.00 & 5.62 & 38 & 0.00 & 6.88 & 39 & 0.00 & 4.33 & 36 & 0.00 \\
\hline 10 & 10 & 7.58 & 37 & 0.00 & 2.65 & 33 & 0.00 & 5.78 & 40 & 0.00 & 3.02 & 35 & 0.00 \\
\hline
\end{tabular}

Table 7: Improvement by LS, using different methods for the starting solution. Caserta instances, $H_{\max }=H+2$. 
addition, the percentage gap between these two values is provided. Percentage gap is computed as $100 \frac{b-a}{b}$, where $b$ is the number of relocations before local search and $a$ is the number of relocations after local search. In the case where not all of the 40 instances belonging to an instance class could be solved, the value in parentheses gives the number of instance which were not solved due to time restrictions. This only happens for the largest instances with $H_{\max }=H+2$. 

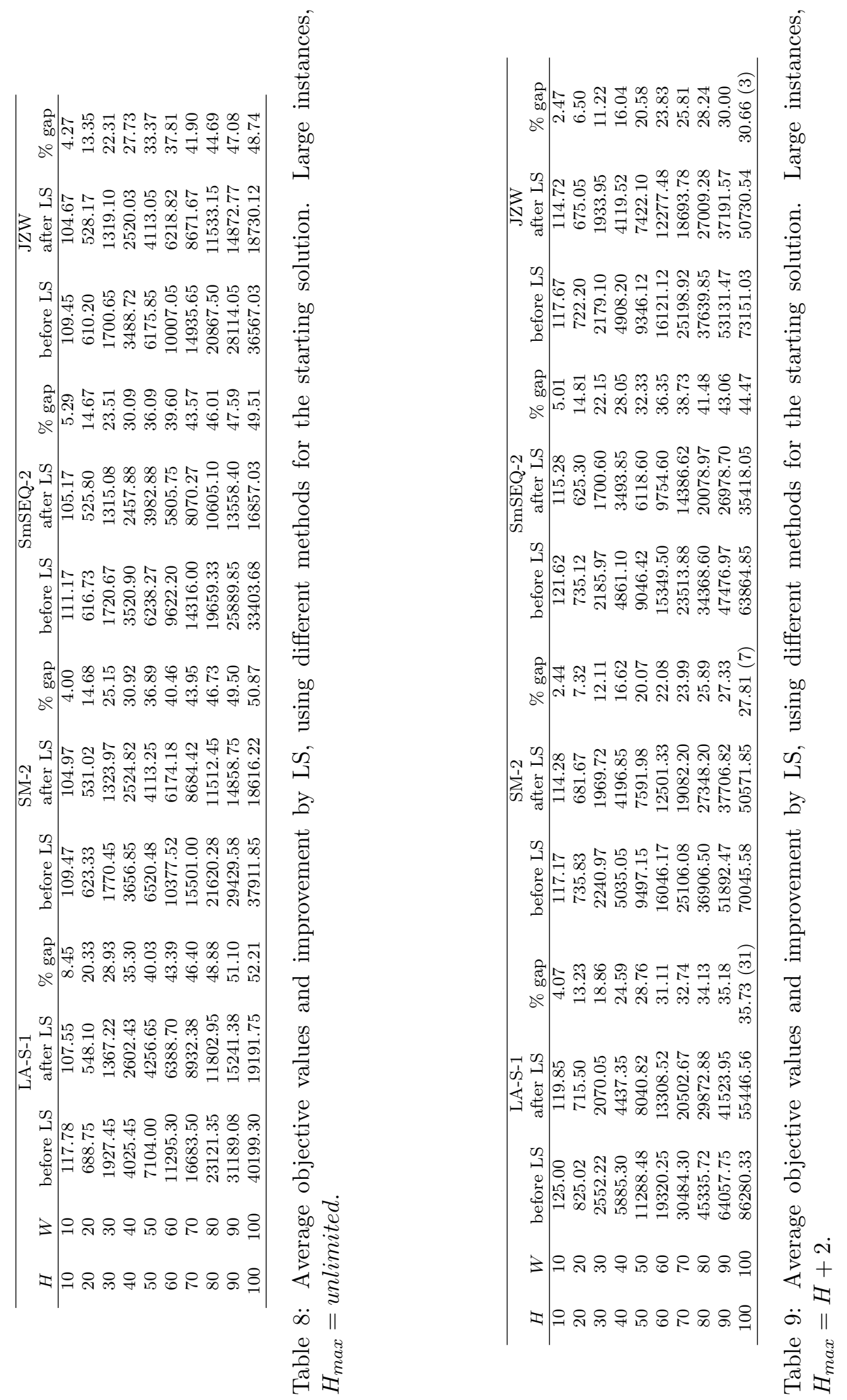


\section{Detailed results for wide instances}

As in the previous appendix, we report average objective values before and after LS as well as the percentage gap between these two values, this time for wide instances. Tables 10 and 11 present these results. 


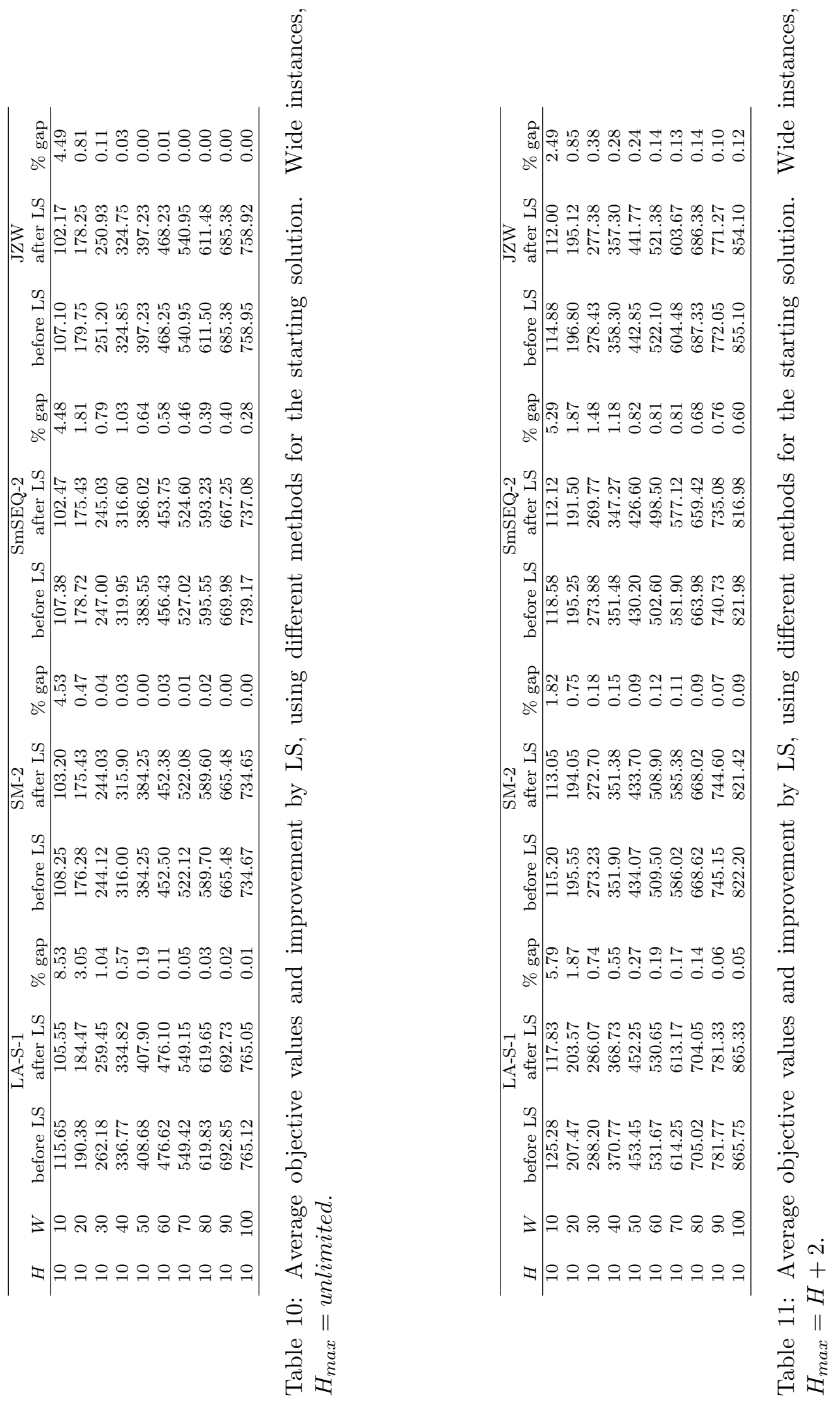

